\title{
Layered Structures of Assembled Imine-Linked Macrocycles and Two- Dimensional Covalent Organic Frameworks Give Rise to Prolonged Exciton Lifetimes
}

\author{
Waleed Helweh, ${ }^{\ddagger \dagger, \S}$ Nathan C. Flanders, ${ }^{\equiv, \dagger, \S}$ Shiwei Wang, ${ }^{\dagger}$ Brian T. Phelan, ${ }^{\S}$ Pyosang Kim,${ }^{\dagger}$ Michael \\ J. Strauss, ${ }^{\dagger}$ Rebecca L. Li, ${ }^{\dagger}$ Matthew S. Kelley, ${ }^{\dagger}$ Matthew S. Kirschner, ${ }^{\dagger}$ Dillon O. Edwards, ${ }^{\dagger}$ Austin P. \\ Spencer, ${ }^{\dagger}$ George C. Schatz, ${ }^{\dagger}$ Richard D. Schaller, ${ }^{\dagger}{ }^{\dagger}$ William R. Dichtel, ${ }^{\dagger *}$ Lin X. Chen ${ }^{\dagger, \S *}$ \\ 'Department of Chemistry, Northwestern University, 2145 Sheridan Road, Evanston, IL, 60208 USA \\ †Center for Nanoscale Materials, Argonne National Laboratory, Argonne, IL 60439, USA. \\ ${ }^{\S}$ Chemical Sciences and Engineering Division, Argonne National Laboratory, Argonne, IL 60439, USA.
}

\begin{abstract}
Ordered organic materials and assemblies have great potential to be tailored to have desirable properties for optoelectronic applications, such as long exciton lifetime and high directional exciton mobility. Framework materials, such as twodimensional covalent organic frameworks (2D COFs), as well as their truncated macrocyclic analogues, are versatile platforms to organize functional aromatic systems into designed assemblies and robust materials. Here we investigate the exciton dynamics in a 2D COF, its corresponding hexagonal macrocycle, and extended nanotubes comprised of stacked macrocycles. The excitonic behavior of these three systems provide an understanding of excitonic processes that occur in the plane of the covalently bonded $2 \mathrm{D}$ macromolecules and between layers of the nanotubes and 2D COF. The nanotube and analogous 2D COF exhibit longer excited-state lifetimes $(\sim 100 \mathrm{ps})$ compared to the individual, solvated macrocycles $(<0.5 \mathrm{ps})$. These differences are attributed to the internal conversion facilitated by the internal motions of the imine linkages which are significantly reduced in the assembled macrocycles in the nanotube and 2D COF sheets in the layered structures. The exciton diffusion processes in the assembled nanotubes and 2D COF systems were characterized by the autocorrelations of the transition dipole moment of the excitons, giving the depolarization time constants for both systems to be $\sim 1$ ps. This work also reveals the anisotropic exciton dynamics related to the in-plane and inter-plane structural factors in these systems. These studies provide guidance for the design of future COF materials, where the longer excited state lifetimes imparted by assembly are beneficial for optoelectronic applications.
\end{abstract}

\section{INTRODUCTION}

Inspired by the natural photosynthetic machinery, in which efficient light-harvesting is achieved by specific interchromophore orientations and distances, recent studies have targeted the synthesis of structurally precise materials or supramolecular assemblies to achieve similar effects. ${ }^{1-4}$ The degree to which these precise structural components dictate functions in energy transfer have been investigated in numerous studies to gain a thorough understanding of excitonic energy migration dynamics in ordered assemblies. 5, 6 The results from these studies will provide design principles for directional and efficient energy transfer in synthetic systems.

Two-dimensional covalent organic frameworks (2D COFs) organize their monomers into crystalline, permanently porous polymer networks. 2D COFs usually form approximately eclipsed, layered structures, providing $\pi$-orbital overlap that might give rise to mobile excitons and other delocalized electronic properties. Because of these characteristics, these materials have been implemented in photovoltaics, ${ }^{7,8}$ chemical sensors, ${ }^{9-11}$ and catalysis. ${ }^{12-14}$ In particular, 2D COFs have gained notable interest because their dimensionality aligns functional aromatic systems, an attribute that has inspired their potential use in optoelectronic devices. ${ }^{15-}$

${ }^{19}$ Furthermore, small interlayer spacings in 2D COFs allow for strong interlayer chromophore coupling - an important attribute for efficient charge and energy transfer. ${ }^{20}$ Despite the promise in these applications, their related electronic properties are challenging to characterize using optical absorption spectroscopy, because of poor optical quality in films resulting from limited solubility. In some cases, they can only be studied as colloids, dispersions or low optical quality thin films, whose tendency to scatter light encumbers accurate measurements on electronic processes as well as eventual integration into devices. Despite these hinderances, previous spectroscopic studies have revealed long-lived charge transfer states and free carrier states, as well as the capability to undergo efficient excitonic transport. $^{21-23}$ However, in the case of triphenylene-based boronate ester-linked COFs, rapid conversion of the $S_{1}$ state to the excimer state significantly shortened the exciton lifetimes of these materials, thereby limiting their overall capacity for long-range energy transport. ${ }^{21}$

In order to investigate exciton behaviors influenced by the local and long-range structural factors, here we characterize photophysical behaviors of an imine-linked 2D COF, a macrocycle (MC) that approximates a single hexagonal unit of the COF, and its assembled, layered columnar nanotube. ${ }^{24-27}$ Taken together, these measurements provide insight into how each component influences the overall electronic properties. The monomers used for MC synthesis are analogues of $\mathrm{COF}$ building blocks, in which one of the reactive moieties is 
replaced by a solubilizing decyloxy side chain. This substitution provides discrete, soluble $\mathrm{MCs}$ that retain chemical and structural similarity to COFs. The MCs assemble into high aspect ratio nanotubes (NTs) upon protonation by a strong acid ${ }^{28}$ resulting in layered structures that approximate the noncovalent interlayer interactions in 2D COFs (Scheme 1). Taken together, these systems enable systematic studies of the exciton dynamics of 2D COFs in comparison to both isolated MCs and assembled NTs.

Such a progression, from MC to NT to 2D COF with increasing dimensions, allows us to capture the structural influence of the exciton properties, such as lifetime, transport dynamics and directionality by ultrafast transient absorption (TA) spectroscopy. This work will describe exciton dynamics in these systems, especially the excited state lifetime upon aggregation. Furthermore, the exciton diffusion along different structural packing directions of these three systems will be characterized by transient absorption anisotropy (TAA). These results demonstrate that proper design of building blocks, chromophores, and their extended structures in COFs can enable rapid energy transfer through their planes, in addition to imine $\mathrm{COF}$ structures prolonging exciton lifetimes, promising effectiveness of COFs and NTs in a number of optoelectronic devices.

\section{METHODS}

Synthesis of DAPB-DiOMe-PDA MC. The macrocycle was synthesized and characterized according to previous procedures $^{26}$ (Scheme 1). A solution of bifunctional arylamine (diaminophenyl benzene [DAPB]) and dialdehyde (2,5dimethoxyterephthalaldehyde [DiOMe-PDA]) were made in 1,4-dioxane $(3.4 \mathrm{~mL})$ in a 4 - $\mathrm{mL}$ closed vial $(30 \mathrm{mM}$ solution of both components). Immediately after adding $\mathrm{CF}_{3} \mathrm{CO}_{2} \mathrm{H}(5 \mu \mathrm{L}$, 0.5 equiv) and shaking, a precipitate formed. The solutions were left to sit without stirring (6 days). The reaction was run for 6 days because shorter reaction times revealed a population of five-membered MCs by MALDI-MS, which disappeared after extended reaction times. $\mathrm{Et}_{3} \mathrm{~N}(0.14 \mathrm{~mL}, 10$ equiv) was added to neutralize the acidic catalyst and the resulting precipitate was isolated by centrifugation. The precipitate was subsequently resuspended, rinsed, and centrifuged with $\mathrm{Et}_{2} \mathrm{O}(3 \times 15 \mathrm{~mL})$, EtOAc $(3 \times 15 \mathrm{~mL})$, hexane $(3 \times 15 \mathrm{~mL})$, and anhydrous acetone $(3 \times 15 \mathrm{~mL})$. The solid was dried under vacuum at room temperature overnight to obtain $44 \mathrm{mg}$ ( $66 \%$ yield) of an orange powder.

Synthesis of DAPB-DiOMe-PDA amorphous polymer. Briefly outlined below is the synthetic scheme for the polymer, which follows a modified version of a previous procedure ${ }^{26}$ (Scheme 1). To synthesize the polymer, identical reaction conditions to the macrocycle were carried out, with the main exception being that tetrahydrofuran was used as the solvent, rather than 1,4-dioxane.

Synthesis of Nanotube: Formation of the NT from the MC was performed using previously reported procedures. ${ }^{28}$ Briefly, solutions of $\mathrm{MC}$ in THF were generated at $100 \mu \mathrm{M}$ concentration and diluted until absorbance was $\sim 0.25$ at 400 $\mathrm{nm}$. $10 \%$ by volume of trifluoroacetic acid (TFA) were added to the MC solution, leading to the rapid formation of the NTs as indicated by a redshift of the absorbance.

Synthesis of TAPB-DiOMe COF colloids Solutions of trisaminophenyl benzene [TAPB] $(3.0 \mathrm{~mL}, 14 \mathrm{mM})$ and 2,5dimethoxybenzene-1,4-dicarbaldehyde (15 mL, $4.2 \mathrm{mM})$ in acetonitrile $(\mathrm{MeCN})$ were prepared separately and combined. A solution of $\mathrm{Sc}(\mathrm{OTf})_{3}(2.0 \mathrm{~mL}, 2.5 \mathrm{mM})$ in $\mathrm{MeCN}$ was added, and then the mixture was shaken briefly by hand to ensure mixing. The combined reaction mixture was held at room temperature for 20 hours. Suspension was then centrifuged for 1 hour, and concentrate was separated from supernatant. Concentrate solvent was evaporated and remaining product was washed 5x with $\mathrm{MeCN}$, and then resuspended in fresh $\mathrm{MeCN}$

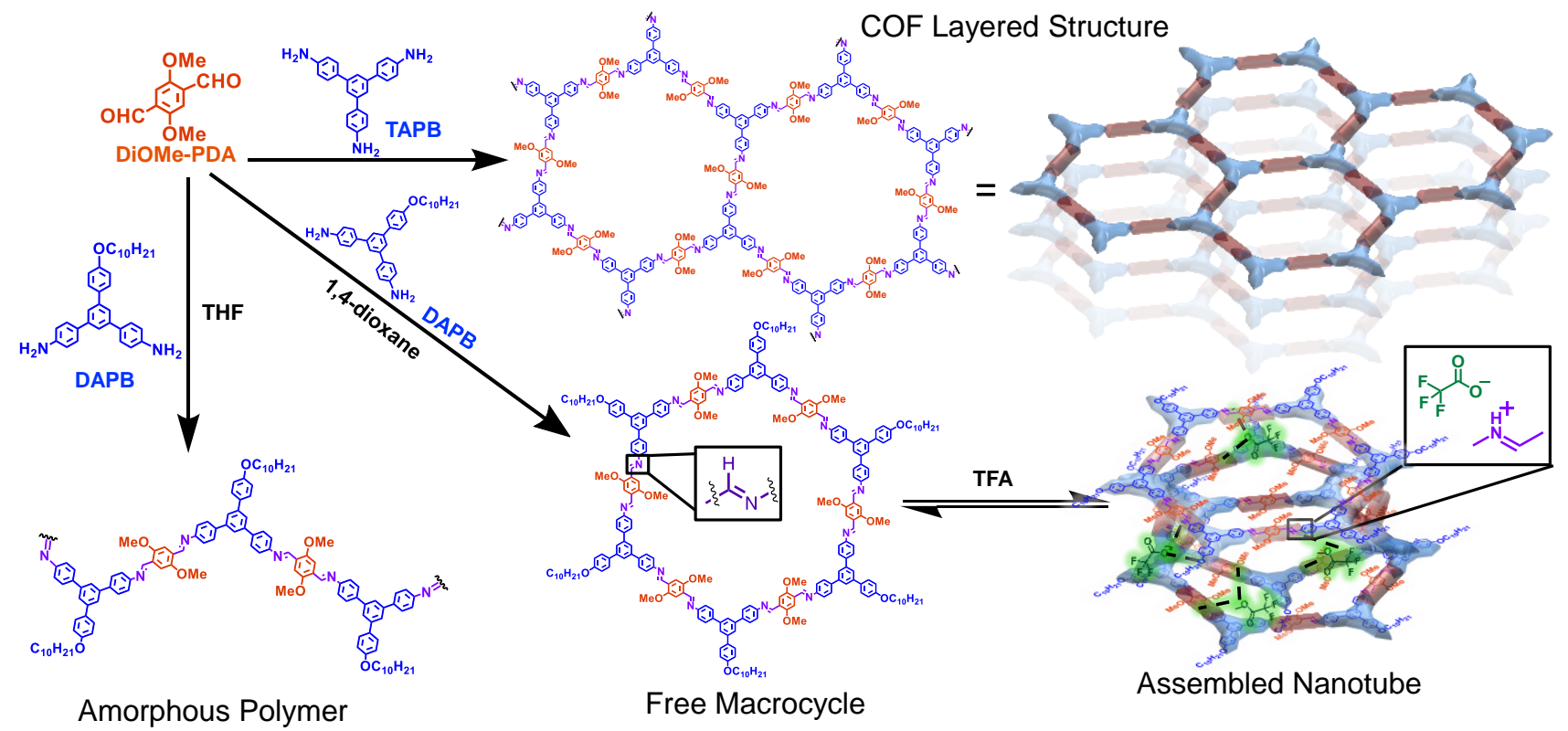

Scheme 1. Structures of the building blocks, TAPB, DAPB and DiOMe-PDA, that comprise the network, the non-hexagonally condensed amorphous polymer, the free imine-linked macrocycle generated from condensation of DAPB and DiOMe-PDA. Addition of large equivalents of TFA result in the assembly of the macrocycles into the corresponding NT. The structure of the $2 \mathrm{D}$ COF generated from condensation of the similar building blocks as the macrocycle and the NT. 
via sonication. Synthesis via this methodology yields COF particles with crystalline domains $\sim 70 \mathrm{~nm}$ in size. Suspension was diluted until its optical density reached $\sim 0.25$ at $400 \mathrm{~nm}$ and was stirred during all measurements. ${ }^{29}$

UV-Vis Spectroscopy. Steady-state spectra of samples diluted with their respective solvents to an optical density of $\sim 0.25$ at $400 \mathrm{~nm}$ in a 1-mm path quartz cuvette were recorded on a Shimadzu UV-3600 Spectrophotometer at room temperature.

Fluorescence Lifetime Measurements. Samples were photoexcited using a $400 \mathrm{~nm}$ pump outputted as the frequencydoubled wavelength of a $35 \mathrm{fs}$, Ti:Sapphire laser with a $2 \mathrm{kHz}$ repetition rate. Photoluminescence decay kinetics were measured by detecting photoluminescence at a $150 \mathrm{~mm}$ spectrograph using a single-photon sensitive streak camera with an instrument response function (IRF) of $\sim 5 \mathrm{ps.}$

Femtosecond and Nanosecond Transient Absorption Spectroscopy. Femtosecond transient absorption (fsTA) measurements were performed using multiple previously described systems. Namely, a Ti:Sapphire regenerativelyamplified laser system (Spectra-Physics MaiTai Oscillator / Spitfire Pro XP amplifier) outputting $830 \mathrm{~nm}$ pulses $(1 \mathrm{kHz}, 100$ fs, $2.6 \mathrm{~mJ}) .^{30,31}$ The samples were excited with $415 \mathrm{~nm}$ pulses $(1 \mu \mathrm{J})$ and probed with broadband continuum pulses (450-800 $\mathrm{nm})$ and the transient spectra were recorded using a Helios transient absorption spectrometer and associated software (Ultrafast Systems). Additionally, other measurements were performed using additional ultrafast laser system as described previously. ${ }^{32,33}$

Nanosecond transient absorption (nsTA) spectroscopy was performed using a previously described Nd:YAG mode-locked laser (EKSPLA PL2210, $500 \mathrm{kHz}, 25 \mathrm{ps}$ FWHM pulses) as the pump and a supercontinuum laser (Leukos STM-1-UV, $1 \mathrm{kHz}$, $600 \mathrm{ps}$ pulses) with white light output as the probe. The samples were excited with $415 \mathrm{~nm}$ pulses $(1 \mu \mathrm{J})$ and probed with the supercontinuum pulses (400-800 $\mathrm{nm}$ ) and recorded using a SP2150i Acton Series spectrograph (Princeton Instruments) coupled with a Spyder3 SG-14 (Teledyne DALSA) CCD camera and laboratory-written software (LabVIEW).

Full details for the fsTA and nsTA procedures can be found in Supplementary Note 1 in the Supporting Information.

Femtosecond Transient Absorption Anisotropy Spectroscopy. Femtosecond two-color transient absorption (TA) experiments were performed using two home-built visible noncollinear optical parametric amplifiers (vis-NOPAs) pumped by $800 \mathrm{~nm}, 35$-fs (FWHM) pulses from a $10-\mathrm{kHz}$ regeneratively-amplified Ti:Sapphire laser (Solstice Ace, Spectra Physics). The pump pulses at $400 \mathrm{~nm}$ were obtained by the second harmonic generation of the fundamental pulse through a $2 \mathrm{~mm}$ thick Type I BBO crystal $\left(\varphi=29.2^{\circ}\right)$, while the probe pulses centered at $\sim 580 \mathrm{~nm}$ with $\sim 100 \mathrm{~nm}$ bandwidth were compressed by a pulse shaper using the multiphoton intrapulse interference phase scan method (MIIPS) with a spatial light modulator (MIIPSBox640, Biophotonic Solutions) resulting in $\sim 10$ fs pulse (FWHM) in a chirp-free spectral region of $500-700 \mathrm{~nm}$. The pump and probe beams were each focused at the sample position using a $25-\mathrm{cm}$ focal length concave mirrors, respectively. All TA measurements were carried out with the pump polarization at the magic angle $\left(54.7^{\circ}\right)$ with respect to the polarization direction of the probe. The time delay between pump and probe pulses was varied using a computer controlled motorized translation stage (ILS300LM, Newport).
For the TA spectra, the pump-on and pump-off spectra were obtained by a chopper operating at $200 \mathrm{~Hz}$ and detected with a spectrograph (Shamrock, Andor) and EMCCD camera (Newton, Andor). The IRF of the experiment was determined to be $\sim 100 \mathrm{fs}$ at $\sim 400 \mathrm{~nm}$ by cross-correlation measurement between the pump and probe pulses using $5 \mu \mathrm{m}$ BBO crystal (United Crystals Inc.). All samples were placed in a $1 \mathrm{~mm}$ quartz cuvette with $\sim 0.5$ optical density around $520 \mathrm{~nm}$.

For the TA anisotropy measurements, the polarization of the probe beam was set to $45^{\circ}$ relative to the vertical polarization of the pump beam. The probe beams parallel $(\|)$ and perpendicular $(\perp)$ to the polarization of the pump beam were separated by a polarizing beam splitter cube and then read out at the same time in two regions (defined as tracks in the Multi-Track mode) of the EMCCD chip.

\section{RESULTS AND DISCUSSION}

Steady-State Optical Characterization of Components. Steady-state UV-Vis spectroscopy of the imine-linked MC reveals a slight red shift of the lowest-energy transition of the linker $\left(\lambda_{\max }=394 \mathrm{~nm}\right)$ upon condensation of the building blocks into the MC $\left(\lambda_{\max }=415 \mathrm{~nm}\right.$, Figure 1a). The lowest energy transition results in an intramolecular charge transfer excitonic state from the DiOMePDA donor to the DAPB acceptor building block (DFT calculations, Supplementary Note 2, Figure S1). ${ }^{34}$ The red shift upon formation of the MC $(0.16 \mathrm{eV})$ is indicative of increased electron delocalization due to the formation of the imine linkages between the two building blocks DAPB and DiOMe-PDA in the MC. ${ }^{35}$ In this case, the $\pi$-electrons are unlikely to delocalize throughout the $\mathrm{MC}$ due to a few components: the polarizability of the imine bond, the presence of meta-linked aromatic systems, as well as the nonplanarity of the MC due to its flexible conformation as reported previously. ${ }^{26,28,36,37}$ Furthermore, an amorphous polymer formed from the same monomers has similar absorption characteristics to those of the MC with an equivalent $\lambda_{\max }$ of 415 $\mathrm{nm}$ (Figure S2). As the polymer would more easily distort in solution as well as having distinct structural components, these results suggest that conjugation is not spread around the $\mathrm{MC}$, and is in fact confined to a single arm of the MC.

When assembled into NTs, a significant red shift (415 nm to $485 \mathrm{~nm}$ ) in the lowest energy transition occurs (Figure 1B). The observed red shift $(0.43 \mathrm{eV})$ can be rationalized by a few phenomena. First, self-assembly flattens the MC - electrostatic attraction in the NT as well as the imine bond polarization caused by its protonation forces the MCs to assemble, planarizing the MCs and leading to slightly increased in-plane conjugation. Along these lines, DFT-calculated absorption spectra (Figure S3) on a truncated model system equivalent of an arm of the hexagonal MC with two TAPB groups bound to a DiOMe-PDA reveal similar trends as observed in the MC and NT, with the flattened model having a red-shifted lowestenergy transition relative to the geometry optimized model that deviates from a perfectly planar conforamtion. Secondly, protonation sequesters the electron lone pair, allowing for the imine to behave as a better electron acceptor and facilitating the CT excitonic state, shrinking the HOMO-LUMO bandgap. This phenomenon has been noted in the case of other imine-based COF's in the presence of acid. ${ }^{38}$ Finally, it has been noted that the nature of the induced aggregation via protonation leads to slip-stacked chromophores such that inter-MC coupling likely has J-type character, a phenomenon known to induce redshifts 

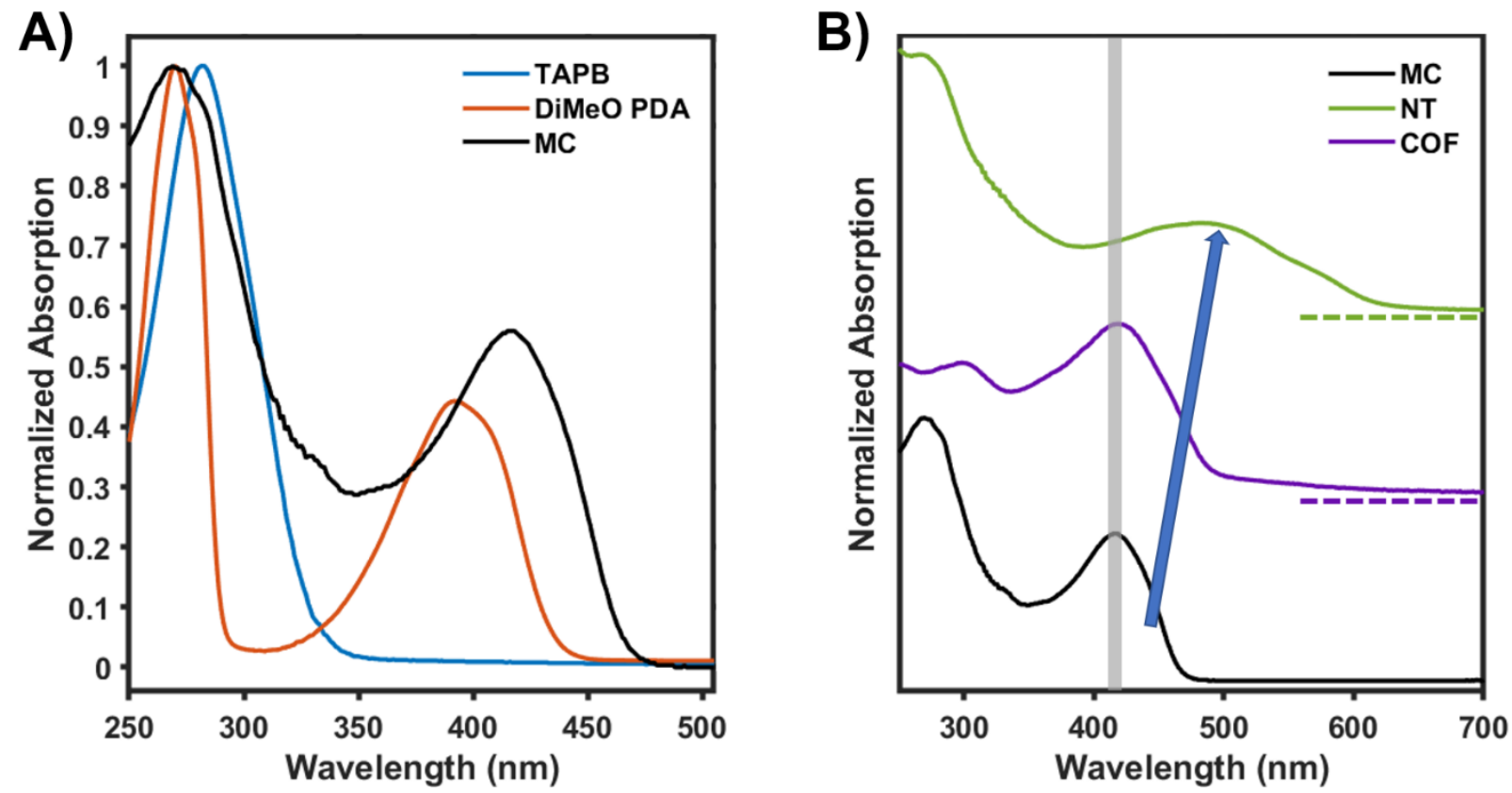

Figure 1. Steady-state optical characterization of the MC, its building blocks, and its derivatives. UV-visible absorption spectra in THF of A) the TAPB building block, the DiOMe-PDA building block, and the corresponding imine-linked MC and B) the MC, its NT derivative formed upon addition of TFA, and a corresponding imine-linked COF with vertical off-sets for display. All spectra are normalized to the greatest absorption and a blue arrow indicates the lowest transition peak energy shift.

in absorbance. ${ }^{39}$ The broadening of the absorption band is in part due to the inhomogeneous nature of the different aggregation states present in the solution as well as mixing between the vibrational states due to electron delocalization. ${ }^{35}$

The lowest-energy absorption peak of the COF is also redshifted relative to the MC $\left(\lambda_{\max }=422 \mathrm{~nm}\right.$, shifted by $\left.0.05 \mathrm{eV}\right)$. However, the NT shows a more significant shift than the COF as well as a longer tail in the low-energy region. These spectra reveal the degree to which the protonation and interlayer chromophore effects come into play. One factor that results in the observed redshift is the difference in solvents. In the case of the $\mathrm{COF}$, the more polar $\mathrm{MeCN}$ solvent would stabilize the polar excited state relative to the THF solvent used for the MC. Additionally, the 2D COF sheets adapt a more planar conformation than the MC due to the stacking, similar to the NT, which would cause a redshift as indicated earlier; however, they have different inter-sheet couplings. As noted by the powder X-ray diffraction (PXRD) pattern, the chromophores in the COF sit cofacially with one another, which does not induce J-type coupling. The primary drivers of the significant redshift seen in the NT, thus are the shrinking of the HOMO-LUMO gap because of the stabilization of the electron-accepting imine as well as the effects of interchromophore coupling through stacking (Figure S1).

In fact, the addition of large equivalents of TFA to the COF colloids results in a similar red-shift to what is observed in the formation of the NT (Figure S4). This confirms the role of lone pair protonation of the nitrogen in the imine bonds in decreasing the HOMO-LUMO bandgap. However, the COF structure reveals a $\lambda_{\max }$ of $510 \mathrm{~nm}, 0.13 \mathrm{eV}$ more than what is observed in the case of the NT. This is likely the result of the more polar solvent stabilizing the excited state as well as the larger extended nature of the COF structure resulting in a greater degree of conjugation. The similarities in the steady-state spectra of the MC and the COF further support the ability for the $\mathrm{MC}$ and its derivatives to serve as effective models for $\mathrm{COF}$ dynamics.

Exciton Dynamics of Free Macrocycle. TA spectroscopy with the photoexcitation of the MC at $415 \mathrm{~nm}$ reveals the emergence of a broad excited-state absorption (ESA) feature from 510 to $760 \mathrm{~nm}$, with the decreased intensity at wavelengths below $500 \mathrm{~nm}$ partially stemming from a ground-state bleach (GSB) feature starting at $460 \mathrm{~nm}$, which was unfortunately outside the white light probe spectral region (Figure 2A). Additionally, a shoulder feature centered at $480 \mathrm{~nm}$ was observed. Kinetic traces of the ESA centered at $630 \mathrm{~nm}$ rapidly decays in $<0.5$ ps (Figure 2B). Conversely, the ESA feature at $480 \mathrm{~nm}$ decays more slowly with a longer lifetime of $2 \mathrm{ps}$.

The broad ESA feature centered at $630 \mathrm{~nm}$ for the MC, immediately being formed, corresponds to $S_{1}$ absorption (Figure 2A) and decays much faster than typical singlet excited states for organic molecules, which are typically on the order of nanoseconds. These short excited-state lifetimes suggest alternative excited-state decay pathways for the MC which are not facile in the other common organic chromophores (Figure 2B). In the case of the isolated MC, the rapid decay of the singlet state likely arises from their flexible bonds in solution which enable internal motions of the groups in the system. As noted in other imine-linked chromophores, the bonding flexibility might enhance the internal conversion route for the excited state or exciton decay to the ground state. ${ }^{40,41}$ The mechanism of this deactivation process is suggested to be caused by the proximity effect imparted on the $n-\pi^{*}$ and $\pi-$ $\pi^{*}$ transitions as the unhindered imine bond gains access to the $\mathrm{S}_{1} / \mathrm{S}_{0}$ conical intersection. ${ }^{42,}{ }^{43}$ Ultimately, the potential energy surface is such that along the reaction coordinate, the excited molecule migrates to said intersection and relaxes to the trans or cis product (Figure 2C). During this process, the double bond is claimed to lose its $s p^{2}$ character and gain $s p^{3}$ character as it approaches the conical intersection. ${ }^{44}$ 

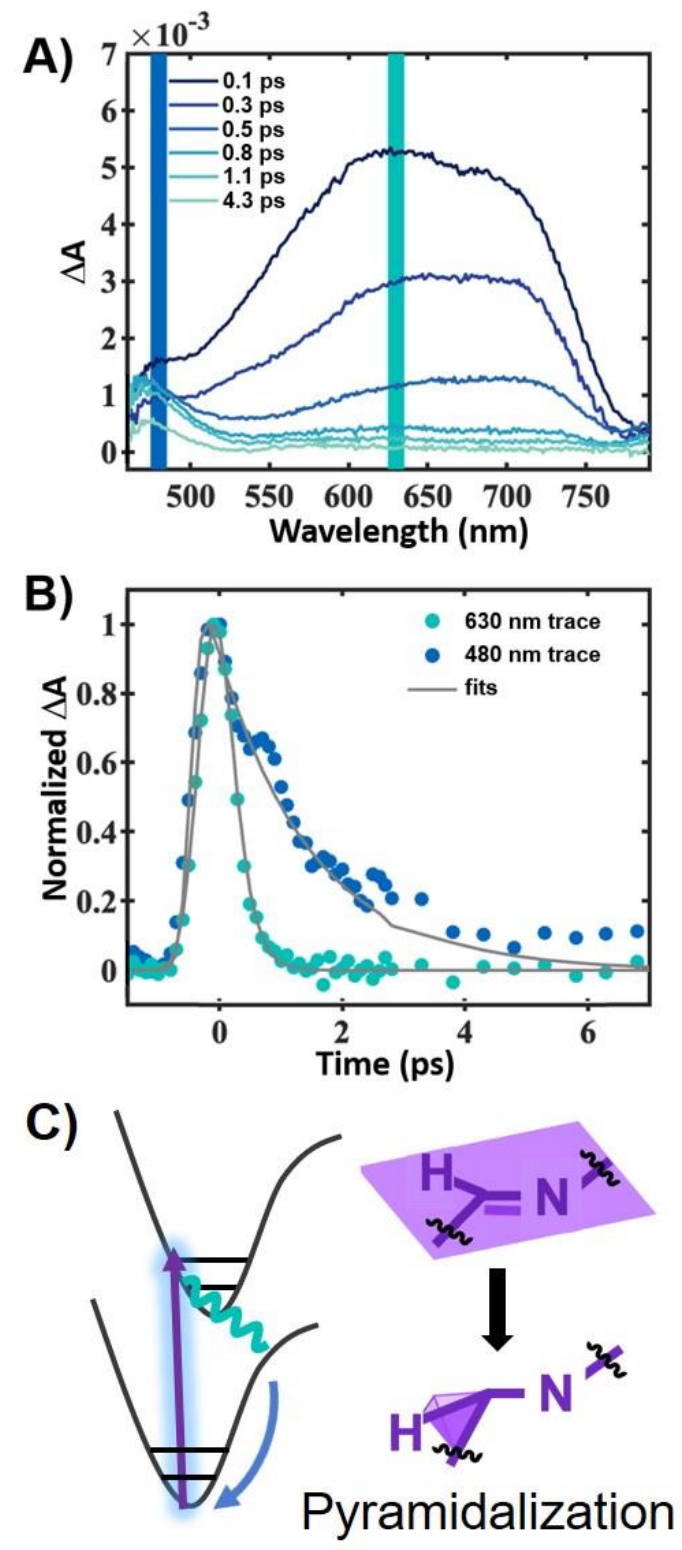

Figure 2. Transient absorption characterization of free macrocycle. A) Transient absorption spectra of free macrocycle with probe wavelengths highlighted at 480 and $630 \mathrm{~nm}$. B) Kinetic trace of macrocycle probed at 480 and $630 \mathrm{~nm}$ revealing divergent kinetics. Trace at $630 \mathrm{~nm}$ reveals rapid deactivation of S1 state. C) Schematic demonstrating kinetic pathway including internal conversion of the excited state to a vibrationally excited ground state which follows slower recovery. Representation of proposed pyramidalization which facilitates internal conversion via conical intersection.

Notably, it is impossible for the imine bonds in the MC to be in the cis form as this causes extreme strain on the cyclic MC structure (Figure S5). This conclusion suggests that only pyramidalization is necessary to approach the $\mathrm{S}_{1} / \mathrm{S}_{0}$ conical intersection and undergo rapid internal conversion - generating the equivalent isomeric product is unnecessary.

The ESA feature at $480 \mathrm{~nm}$ is redshifted from the ground state absorption and exhibits a derivative line shape at the onset of the ground state absorption position (Figures 2A and S6). As mentioned above the MC is believed to rapidly decay to the ground state via the conical intersection accessed by the imine bond. Thus, the induced absorption at $480 \mathrm{~nm}$ is likely the result of vibrationally excited ground state absorption. As the excited state deactivates to the ground state via internal conversion, it enters into a high energy manifold of vibrational states within the ground state. As this population has a smaller energy transition to the excited state due to occupying a higher energy vibrational state, its absorption is redshifted relative to that of the ground state absorption. This "hot" ground state feature also rapidly decays (2 ps), though still more slowly than the $680 \mathrm{~nm}$ ESA, likely resulting from vibrational cooling (Figure 2B). We speculate that the rise of this ESA feature would occur in conjunction with the decay of the ESA feature at $630 \mathrm{~nm}$; however, both processes occur too rapidly to be resolved by the instrument's capabilities. To further confirm the idea of this feature being associated with a vibrationally excited ground state, the position of the absorption feature was determined at each time point by converting the spectral axis to energy and fitting to a Gaussian line shape to determine the peak position (Figure S7). These results reveal that the maximum position of this feature undergoes a hypsochromic shift with an approximate time scale of $2 \mathrm{ps}$. As the vibrationally excited ground state cools, the absorption corresponding to $S_{0}-S_{1}$ transition requires more energy, which is reflected in the observed blue shift.

The amorphous polymer exhibits similar excited state characteristics as the free MC (Figure S8), suggesting similar excited state characteristics to the free MC. Probing at equivalent wavelengths 480 and $630 \mathrm{~nm}$ reveal almost identical responses to the MC with an IRF limited lifetime of $>0.5 \mathrm{ps}$ at $630 \mathrm{~nm}$ and a short $\sim 3 \mathrm{ps}$ component at $480 \mathrm{~nm}$. Also, decreased scattering relative to the MC allows the GSB feature to be more easily resolved at $430 \mathrm{~nm}$ revealing rapid $(>0.5 \mathrm{ps}$ ) recovery of the ground state, via internal conversion coincident with the ESA feature at $600 \mathrm{~nm}$. As the polymer is chemically similar in its imine bond to the $\mathrm{MC}$, it is also suggested to undergo internal conversion via the pyramidalization of the imine bond, and the rapid decay is in fact observed.

Effect of Assembly on Fluorescence Dynamics. Timeresolved photoluminescence studies of the free MC at various temperatures were performed to further investigate the effect of restricting motion on the excited state dynamics. As the temperature is decreased from $333 \mathrm{~K}$ to $80 \mathrm{~K}$, the emission spectrum of the free MC narrows (Figure 3A), which is consistent with narrower conformational disorder in the molecules. The excited state lifetimes also increase as the temperature is decreased (Figure 3B). Strong temperature dependence of the excited state dynamics has been observed with an instrument response-limited $<6$ ps decay at $333 \mathrm{~K}$ increasing to bi-exponential decay upon cooling to $80 \mathrm{~K}$ with a 20 ps time constant and an additional time constant of about 120 ps as well as an offset feature present for the remainder of the experiment. This rapid deactivation of the excited-state population is suggested to result from non-radiative internal conversion pathways provided by the imine bond. ${ }^{40,41,45}$ Roomtemperature fluorescence studies of small molecules with imine bonds show quenching due to the internal conversion and rapid excited-state decay lifetimes. ${ }^{40,41}$ In such systems, when the temperature of the system is decreased, there is a drastic increase in the relative fluorescence quantum yield as well as an increased excited-state lifetime. Therefore, reducing the degrees of freedom of the molecule by decreasing the extent of imine bond pyramidalization hinders the rapid internal 

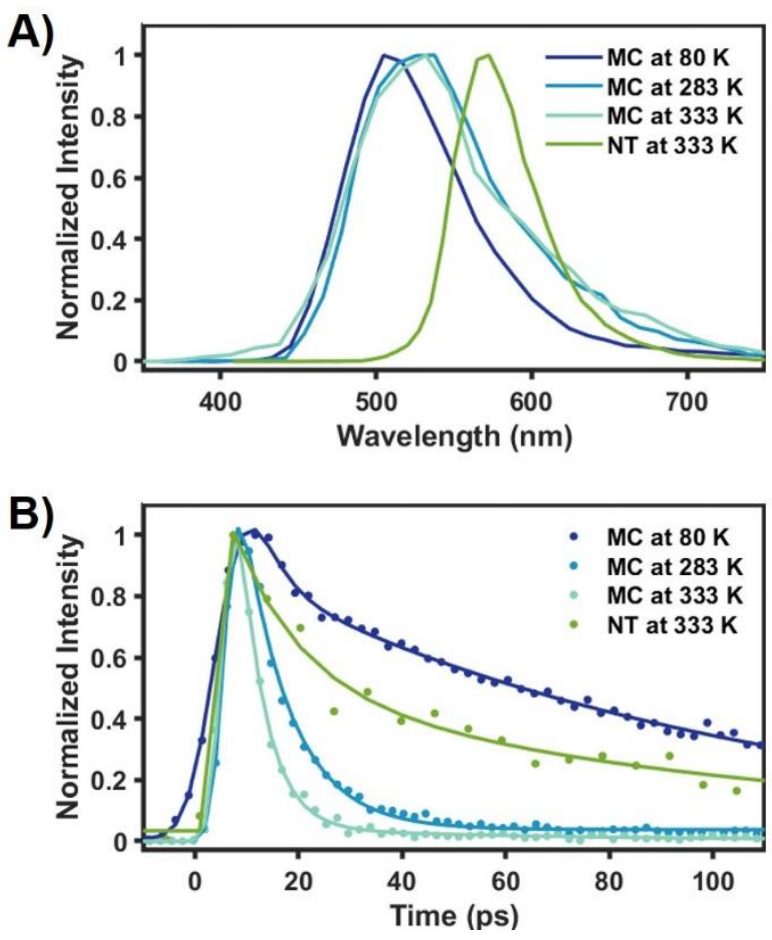

Figure 3. Time-resolved photoluminescence of the macrocycle and nanotube following photoexcitation at $400 \mathrm{~nm}$. A) Spectral cross-sections of the macrocycle at $80 \mathrm{~K}, 283 \mathrm{~K}, 333 \mathrm{~K}$, and the nanotube formed after addition of the acid (at $333 \mathrm{~K}$ ). B) Kinetic traces at maximum photoluminescence intensity revealing longer lifetimes at colder temperatures and after formation of the nanotube.

conversion deactivation pathways and thereby extends the excited-state lifetime. Resultantly, the relative quantum yield of the fluorescence should increase due to the longer times over which to fluoresce. Indeed, a relative quantum yield increase of around two orders of magnitude is observed upon formation of the nanotube structure (Figure S9).

The emission spectrum of the NT shows a red shift from that of the free MC, consistent with the absorption spectra of both species (Figure 3A). The kinetic trace of the NT emission at $333 \mathrm{~K}$ reveals a significantly longer lifetime compared to its MC counterpart at $333 \mathrm{~K}$. In fact, the rapid component of the NT has a lifetime of about 13 ps with a second longer time component beyond the detection time window of the experiment (around $95 \mathrm{ps).} \mathrm{Observing} \mathrm{the} \mathrm{fluorescence} \mathrm{on} \mathrm{a}$ longer time scale reveals the presence of a weak offset feature that is beyond the window that can be probed (Figure S10). The similarity in its spectral profile to early time points infers that it comes from an identical state. This result can be rationalized by looking at the structure of the NT. Since the protonation forces assembly into NTs and makes the structure more rigid, the deactivation pathway allowed by pyramidalization of the imine bond (similar to the effect of decreasing temperature) would likely be drastically reduced, ${ }^{46}$ resulting in an increased excitedstate lifetime.

Exciton Dynamics of Assembled Nanotube and COF. TA spectroscopy was also performed on the assembled NT structure as well as the corresponding COF in solution. The TA signals of the NT revealed a broad ESA feature from 550 to 800 $\mathrm{nm}$, similar to that in the MC, but overlaid with a GSB feature at $\sim 560 \mathrm{~nm}$, which matches with the ground-state absorption (Figure 4A). For the COF, a similarly broad ESA feature peaking at $600 \mathrm{~nm}$ was observed, slightly blue shifted relative to the NT (Figure 4B). Low probe intensity below $500 \mathrm{~nm}$, due to light scattered by the COF sample obscured the GSB feature, but the onset of the GSB feature was observed starting at $<500$ $\mathrm{nm}$, significantly blue-shifted relative to the NT due to the significantly blue-shifted steady-state absorption.

The traces and spectra were fit using a global fitting procedure. Namely, the kinetics were fit at three wavelengths $(580,650,720 \mathrm{~nm})$ to a biexponential decay model as well as a long-lived offset component. The resultant decay features had varied amplitude at different wavelengths revealing the resulting decay-associated spectra. The TA spectra of the free $\mathrm{MC}$, the NT and COF all exhibit broad ESA features spanning much of the visible spectral region. This ESA feature is likely to arise from the $S_{1}$ state due to its direct excitation. The broad nature of the ESA indicates a wealth of different discrete conformations that each feature could occupy allowing for inhomogeneity in the excitonic structure. This feature decayed monotonically with no spectral change, demonstrating no discernible change in the state distribution.

Observing the kinetics of the NT and COF at $630 \mathrm{~nm}$ reveals similar kinetic traces for both species (Figure 4C). The two kinetic traces were fit for both femtosecond TA data and nanosecond TA data. Fitting of the decays reveals ESA decays occurring on three time scales for both species. The first, very rapid decay occurs with a lifetime of $5.3 \mathrm{ps}$ for the NT and 4.3 ps for the COF and the second decay has lifetimes of 51 and 85 ps for the NT and COF, respectively. The signal did not return to the baseline within the time range measured in the fsTA experiments for both the NT and COF. Longer time nsTA spectroscopy resolved a lifetime of $12.5 \mathrm{~ns}$ for that feature in both the NT and COF (Figure S11). Global analysis of the femtosecond dynamics revealed the absorption features of all species. In this case all decay features, as well as the offset, are resultant from similarly broad ESA features across the spectrum (Figure S12). As the rapid component of the NT decay (5.3 ps) is consistent with the rapid decay observed in the time resolved fluorescence measurement (Figure S13), both features likely result from the initially excited $\mathrm{S} 1$ state.

Furthermore, the longer component in the time-resolved fluorescence matches with the long-lived decay component from the $S_{1}$ ESA feature revealing that all decays arise from the $\mathrm{S}_{1}$ state. In particular, the rapid decay component is suggested to be due to vibrational cooling of the initial excitation, happening on the timescale of $<15 \mathrm{ps}$. The longer-lived feature for both the NT and COF ( $51 \mathrm{ps}$ and $85 \mathrm{ps)} \mathrm{likely} \mathrm{represents} \mathrm{the}$ population decay, still arising from the same luminescent state. Furthermore, the offset feature (Figures 4A and 4B) is hypothesized to arise from a slower structural change following photoexcitation that traps a small fraction of the excited state population. Consequently, the overall lifetime of the $S_{1}$ state of the MC chromophore increases from $<0.5$ ps to $\sim 100$ ps upon aggregation and inhibition of the imine bond pyramidalization, a significant prolonging of the excited state lifetime. This inhibition is demonstrated to occur via three routes in this discussion, one the forced assembly via strong electrostatic interactions in the NT, the COF structures which are aggregated via Van der Waals forces, and finally via freezing of the structures at $80 \mathrm{~K}$. This large increase in the excited-state lifetime allows for more time through which excitonic 

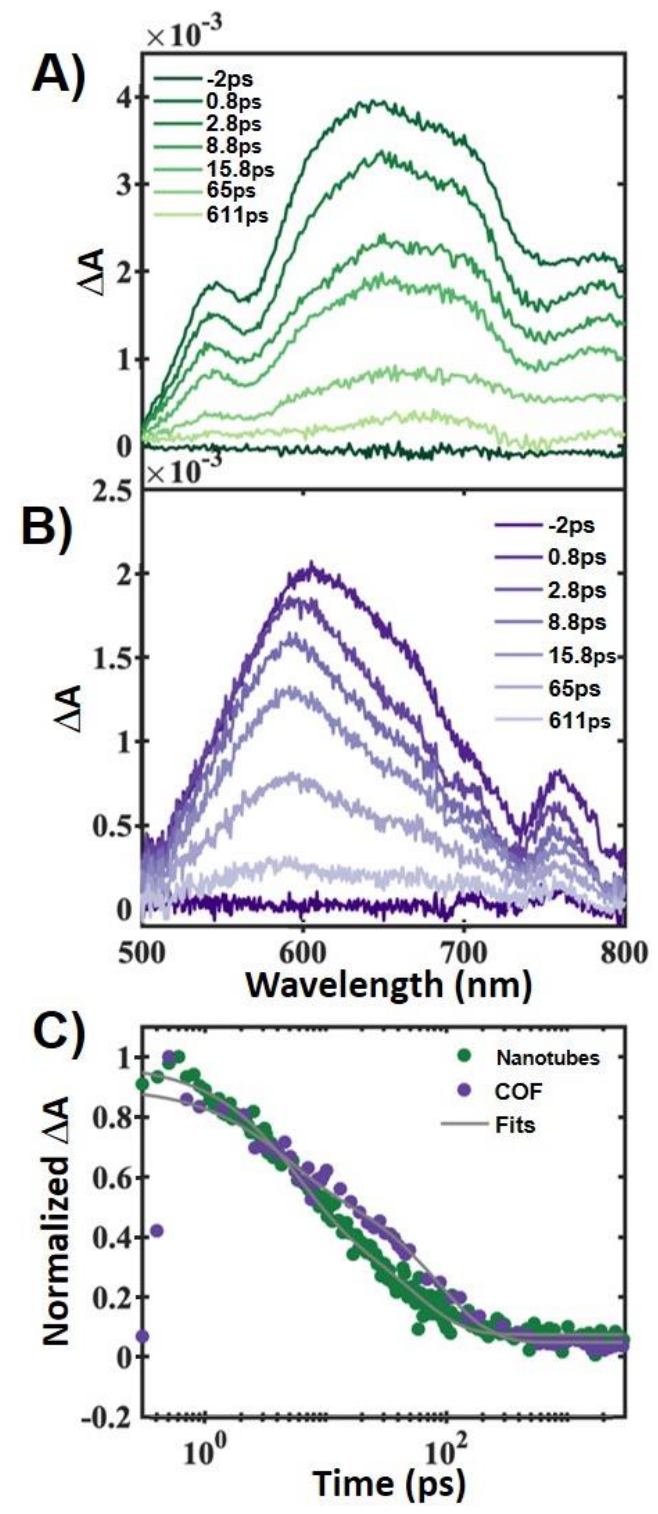

Figure 4. Transient absorption characterization of assembled nanotube and COF. A) Transient absorption spectra of nanotube revealing broad excited state absorption interspersed with ground state bleach $(550 \mathrm{~nm})$ and spontaneous emission troughs $(760 \mathrm{~nm})$. B) Transient absorption spectra of COF also revealing broad excited state absorption interspersed with ground state bleach $(<500 \mathrm{~nm})$ and spontaneous emission troughs $(780 \mathrm{~nm}) . C)$ Kinetic traces of the nanotube and COF samples probed at $630 \mathrm{~nm}$, revealing similar decay kinetics of the excited state and increased excited state lifetimes relative to that of the unassembled macrocycle.

processes can occur, greatly increasing the effectiveness of this material for optoelectronic properties which necessitate longer time scales.

Excitonic Mobility in Nanotubes and COF. To explore the exciton transport dynamics in NTs solutions and COF suspensions power dependent TA was employed (Figure S14). By increasing the fluence of the excitation pulse raises the excited state population to a threshold in which the mobile excitons can undergo exciton annihilation. Only if the excitons are mobile can this process occur, confirming the capability of exciton transport in these materials. ${ }^{21}$

Furthermore, TA anisotropy was employed for further insight into the dimensionality of exciton transport. In this case, samples were excited with a horizontally polarized laser pulse to preferentially excite chromophores with their transition dipoles aligned in that axis to create anisotropically oriented distributions in the ground and excited states. Then the transmitted probe pulse signals after the sample were collected as a function of the pump-probe delays at parallel and perpendicular polarization directions with respect to the pump pulse polarization (see Methods). The resulting TA anisotropy is defined as

$$
r(t)=\frac{I_{\|}(t)-I_{\perp}(t)}{I_{\|}(t)+2 I_{\perp}(t)}
$$

where $I_{\|}(\mathrm{t})$ and $I_{\perp}(\mathrm{t})$ are transient absorption signals at parallel and perpendicular polarization directions, respectively, relative to the polarization direction of the pump pulse. At $630 \mathrm{~nm}$ where the NT S1 state absorbs, $\mathrm{I}_{\|}(\mathrm{t})$ only decays from the initial value at delay time zero, whereas $\mathrm{I}_{\perp}(\mathrm{t})$ rises and then decays (Figure $\mathrm{S} 15$ ).

Considering that excitons initially have a preferred dipole orientational distribution aligned with the excitation polarization, ${ }^{49}$ any change in that initial preferred polarization will be reflected in the differences in intensity of the probe beam polarizations (Equation 1). The initially excited dipole can depolarize through different mechanisms, the most prominent of which are rotational depolarization or energy transfer. In the case of rotational depolarization, the initially excited molecule will depolarize the excited state via rotation of the entire molecule, or at least a segment encompassing the dipole, in the solution. Alternatively, depolarization can occur through energy transfer. As the exciton moves through the material, if the orientation of the transition dipole changes from the initial excitation, it will result in an anisotropy decay within the time window defined by the exciton lifetime. While energy transfer is heavily sample-dependent, hopping between sites typically happens on the order of a few ps. ${ }^{50,51}$

In the case of the NT, $\mathrm{r}(\mathrm{t})$ decay kinetics are consistent across the probe spectrum. The normalized $[\mathrm{r}(\mathrm{t}) / \mathrm{r}(0)]$ anisotropy signal at $630 \mathrm{~nm}$ reveals that the initial excitation depolarizes via two different time constants, 1.34 ps and 8.75 ps (Figure 5A). Additionally, the initial excitation of the COF reveals depolarization rates consistent across the probe spectrum, with decays occurring with a single rate of $1.12 \mathrm{ps}$. Although the exact details for the transition dipole are still unclear, they likely align along a single edge of the hexagon due to the limited redshift upon condensation discussed earlier. For this reason, we will only explore the nature of the depolarization dynamics in this report. In the case of the COF, the $\mathrm{x}$-ray diffraction pattern reveals that the TAPB-DiOMe COF crystallizes in P6 structures, and as such, the inter-planar orientation of transition dipoles are entirely eclipsed and parallel (Figures S16 and S17). ${ }^{29}$ Thus, if energy transfer occurs purely through the stacks of the COF, it would not result in depolarization of the initial excitation (Figure 5C). Conversely, if energy transfer occurs between adjacent chromophores along the plane axis of the 2D sheet, the chromophores are held at different relative angles resulting in depolarization of the initial excitation (Figure 5C). It is also possible that energy transfer occurs via both pathways, but this mechanism would be indistinguishable via this method and would still ultimately 
A)

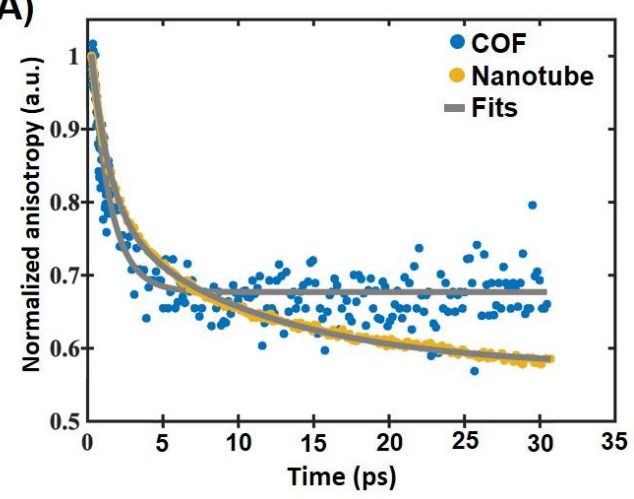

B)

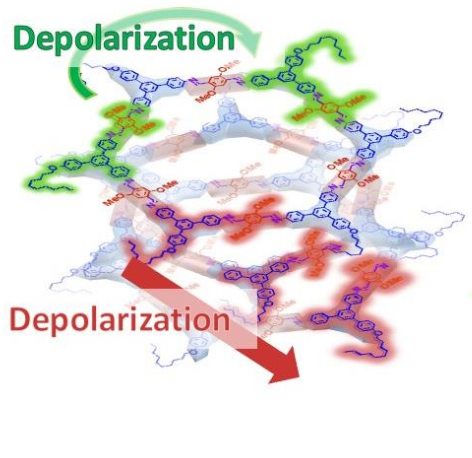

C)

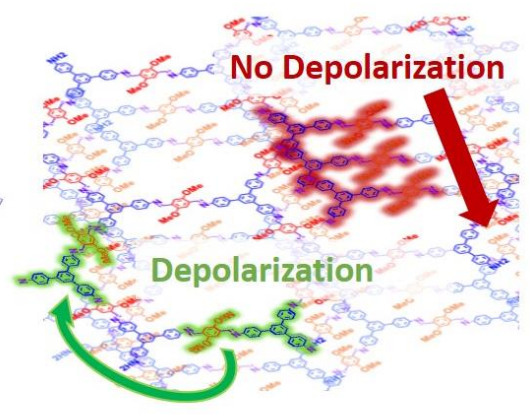

Figure 5. Exciton dynamics via time resolved anisotropy in nanotubes and COF. A) Transient absorption anisotropy kinetics of nanotube probed at $630 \mathrm{~nm}$ revealing two depolarization kinetics. B) Schematic of nanotube demonstrating both intermolecular energy transfer and intramolecular energy transfer. Both forms can lead to depolarization. C) Schematic of COF depolarization scheme showing that energy transfer through stacks would not result in depolarization.

result in the same angle of depolarization as entirely in-plane energy transfer. Thus, the 1.12 ps depolarization of $\mathrm{r}(\mathrm{t})$ in $\mathrm{COFs}$ reflects energy transfer along the plane of the sheets, demonstrating that energy transfer in the plane of the $2 \mathrm{D}$ sheets of this COF occurs rapidly.

Similar to the COF, the NT has a hexagonal structure albeit without the 2D intraplanar extension of the network. As such, energy transfer around the hexagonal structure in the NT will likewise result in depolarization of the initial excitation. However, in the NT, the interplanar chromophores organize in such a way that they are not entirely eclipsed but are in fact slightly offset in relative angle to one another (Figure 5B). Thus, intermolecular energy transfer along the long axis of the NT could simultaneously occur through nearly parallel stacked structure which results in nearly no depolarization. Considering that the exciton anisotropy decay time constants for both the NT and COF are similar (1.3 and 1.1 ps respectively), the exciton hopping is dominant via intraplanar processes within the constituent MCs that comprise the NT. Interestingly, the COF exhibits a slightly faster depolarization rate which may result from the additional sites available for energy transfer within the plane of the $2 \mathrm{D}$ network. Thus, as these two rates are very similar, the longer $8.8 \mathrm{ps}$ depolarization rate in the case of the NT results from interplanar energy transfer along the long axis of the NT.

Previous reports have related the angle between hopping sites as well as the depolarization rate to the hopping rate itself. ${ }^{49,51,52}$ As the offset $\sigma$ (Figure S18) between each MC in the NT covers a broad range of angles, determining specific hopping rates is non-definitive. As such, a plot comparing the angle to the hopping rate demonstrates that angles going from $2^{\circ}$ to $30^{\circ}$ can result in hopping rates that vary from $100 \mathrm{fs}$ to 10 ps depending on the relative angle, $\sigma$, between chromophores, suggesting even at the limit of largest angle, rapid energy transfer is occurring through the NT (Figure S19).

These two results taken together reveal energy transfer occurs both around the ring as well as through the NT itself. While it is not possible to reveal interplanar energy transfer in the COF via TAA, coupling the results which reveal the transfer rates within the plane with future studies on using excitonexciton annihilation will help to extract the two distinct rates of energy transfer in the two different directions. These results inform future design of COF structures for use as optoelectronic materials.

\section{CONCLUSION}

The exciton dynamics of a series of model compounds related to an imine-linked $\mathrm{COF}$ were investigated. We compare the monomer building blocks to the hexagonal MC, to the NT comprised of stacked macrocycles, as well as to the COF structure to reveal important photophysical distinctions among these systems. These investigations reveal that the isolated macrocycles have an excited state lifetime of $<0.5 \mathrm{ps}$, as they rapidly dissipate excited-state energy likely via internal conversion to the ground state driven by pyramidalization of the $s p^{2}$ hybridized carbon in the imine bonds. In the case of the assembled structures such as NTs or the COF, the conical intersection that accelerates internal conversion is no longer accessible, resulting in a prolonged exciton lifetime up to tens of nanoseconds. This long excited state lifetime improves the probability for exciton to diffuse in longer distances and the potential applications involving chemical transformation and sensing. To explore further the degree to which these materials would be effective for use in such optoelectronic applications, energy transfer dynamics of the assembled materials were taken to confirm for the first time (to the best of our knowledge) that energy transfer along the two dimensional axis of the COF sheets can occur, with a depolarization time constant of $1.1 \mathrm{ps}$. Energy transfer through the stacks of macrocycles in the NT is also detected, demonstrating the promise of COF materials for energy transfer-based applications. This study guides design principles for the use of these materials in optoelectronic devices by inferring the dimensionality of energy transfer. Future studies can further elucidate the role structure plays in the excitonic properties of these materials.

\section{ASSOCIATED CONTENT}

\section{Supporting Information}

The Supporting Information is available free of charge on the ChemRxiv website. Further Detail regarding exciton dynamics characterizations etc.

\section{AUTHOR INFORMATION}

\section{Corresponding Authors}

* Lin X. Chen, 1-chen@ @orthwestern.edu, 1chen@anl.gov

*William R. Dichtel, wdichtel@northwestern.edu

\section{Author Contributions}


$\equiv$ These authors contributed equally. The manuscript was written through contributions of all authors. All authors have given approval to the final version of the manuscript.

\section{ACKNOWLEDGMENT}

This material is based upon work supported by the U.S. Department of Energy, Office of Science Graduate Student Research (SCGSR) program. The SCGSR program is administered by the Oak Ridge Institute for Science and Education (ORISE) for the DOE under contract number DE-SC0014664. We acknowledge the Army Research Office for a Multidisciplinary University Research Initiatives (MURI) award under grant number W911NF-15-10447. A part of the work used the Advanced Photon Source and Center for Nanoscale Materials, both are user facilities operated for the DOE Office of Science by Argonne National Laboratory under Contract No. DE- AC0206CH11357. This work has also made use of the IMSERC, EPIC, and Keck II facility of NUANCE Center at Northwestern University, which has received support from the Soft and Hybrid Nanotechnology Experimental (SHyNE) Resource (NSF ECCS-1542205), the MRSEC program (NSF DMR1720139) at the Materials Research Center, the Keck Foundation, the State of Illinois and International Institute for Nanotechnology (IIN). W. H., N.C.F, M.S.K., G.C.S. and L.X.C. are partially supported (TA and theory work) by Basic Energy Science, CBG Division, US Department of Energy through Argonne National Laboratory under Contract No. DE-AC02-06CH11357. S.W. (synthesis) is partially supported by the Northwestern University and Weinberg College Undergraduate Research Grants. M.J.S was supported by the National Science Foundation (NSF) through the Graduate Research Fellowship Program (GRFP) under Grant No. (DGE-1842165). M.J.S. is partially supported by the Ryan Fellowship and the International Institute for Nanotechnology.

\section{ABBREVIATIONS}

COF, Covalent Organic Framework; MC, Macrocycle; NT, Nanotube; DiOMe PDA, 2,5-dimethoxyterepthalaldehyde; TAPB, 1,3,5-trisaminophenylbenzene; DAPB, alkoxyterminated diaminophenylbenzene; ESA, Excited state absorption; SE, Spontaneous emission; TA, Transient Absorption; XRD, X-ray diffraction.

\section{REFERENCES}

1. Logsdon, J. L.; Hartnett, P. E.; Nelson, J. N.; Harris, M. A.; Marks, T. J.; Wasielewski, M. R., Charge Separation Mechanisms in Ordered Films of Self-Assembled Donor-Acceptor Dyad Ribbons. Acs Appl Mater Inter 2017, 9 (39), 33493-33503.

2. Sengupta, S.; Ebeling, D.; Patwardhan, S.; Zhang, X.; von Berlepsch, H.; Bottcher, C.; Stepanenko, V.; Uemura, S.; Hentschel, C.; Fuchs, H.; Grozema, F. C.; Siebbeles, L. D. A.; Holzwarth, A. R.; Chi, L. F.; Wurthner, F., Biosupramolecular Nanowires from Chlorophyll Dyes with Exceptional Charge-Transport Properties. Angew Chem Int Edit 2012, 51 (26), 6378-6382.

3. Nakamura, Y.; Aratani, N.; Osuka, A., Cyclic porphyrin arrays as artificial photosynthetic antenna: Synthesis and excitation energy transfer. Chem. Soc. Rev. 2007, 36 (6), 831-845.

4. Kelley, R. F.; Lee, S. J.; Wilson, T. M.; Nakamura, Y.; Tiede, D. M.; Osuka, A.; Hupp, J. T.; Wasielewski, M. R., Intramolecular energy transfer within butadiyne-linked chlorophyll and porphyrin dimer-faced, self-assembled prisms. J. Am. Chem. Soc. 2008, 130 (13), 4277-4284.

5. Cheng, Y. C.; Fleming, G. R., Dynamics of Light Harvesting in Photosynthesis. Annu. Rev. Phys. Chem. 2009, 60, 241-262.

6. Cleary, L.; Chen, H.; Chuang, C.; Silbey, R. J.; Cao, J. S., Optimal fold symmetry of LH2 rings on a photosynthetic membrane. $P$ Natl Acad Sci USA 2013, 110 (21), 8537-8542.

7. Xu, B.; Li, S. C.; Jiao, H.; Yin, J.; Liu, Z. G.; Zhong, W. Y., A two-dimensional quinazoline based covalent organic framework with a suitable direct gap and superior optical absorption for photovoltaic applications. J Mater Chem A 2020, 8 (7), 3865-3871.

8. Cox, J. M.; Mileson, B.; Sadagopan, A.; Lopez, S. A., Molecular Recognition and Band Alignment in 3D Covalent Organic Frameworks for Cocrystalline Organic Photovoltaics. J Phys Chem C 2020, 124 (17), 9126-9133.

9. Kulkarni, R.; Noda, Y.; Barange, D. K.; Kochergin, Y. S.; Lyu, P. B.; Balcarova, B.; Nachtigall, P.; Bojdys, M. J., Real-time optical and electronic sensing with a beta-amino enone linked, triazinecontaining 2D covalent organic framework. Nature Communications 2019, $10,3228$.

10. Wu, X. W.; Han, X.; Xu, Q. S.; Liu, Y. H.; Yuan, C.; Yang, S.; Liu, Y.; Jiang, J. W.; Cui, Y., Chiral BINOL-Based Covalent Organic Frameworks for Enantioselective Sensing. J. Am. Chem. Soc. 2019, 141 (17), 7081-7089.

11. Peng, Y. W.; Huang, Y.; Zhu, Y. H.; Chen, B.; Wang, L. Y.; Lai, Z. C.; Zhang, Z. C.; Zhao, M. T.; Tan, C. L.; Yang, N. L.; Shao, F. W.; Han, Y.; Zhang, H., Ultrathin Two-Dimensional Covalent Organic Framework Nanosheets: Preparation and Application in Highly Sensitive and Selective DNA Detection. J. Am. Chem. Soc. 2017, 139 (25), 8698-8704.

12. Xiang, Z. H.; Xue, Y. H.; Cao, D. P.; Huang, L.; Chen, J. F.; Dai, L. M., Highly Efficient Electrocatalysts for Oxygen Reduction Based on 2D Covalent Organic Polymers Complexed with Nonprecious Metals. Angewandte Chemie-International Edition 2014, 53 (9), 2433-2437.

13. Lin, C. Y.; Zhang, D. T.; Zhao, Z. H.; Xia, Z. H., Covalent Organic Framework Electrocatalysts for Clean Energy Conversion. Adv. Mater. 2018, 30 (5), 1703646

14. Jin, E. Q.; Lan, Z. A.; Jiang, Q. H.; Geng, K. Y.; Li, G. S.; Wang, X. C.; Jiang, D. L., 2D sp(2) Carbon-Conjugated Covalent Organic Frameworks for Photocatalytic Hydrogen Production from Water. Chem-Us 2019, 5 (6), 1632-1647.

15. Evans, A. M.; Parent, L. R.; Flanders, N. C.; Bisbey, R. P.; Vitaku, E.; Kirschner, M. S.; Schaller, R. D.; Chen, L. X.; Gianneschi, N. C.; Dichtel, W. R., Seeded growth of single-crystal twodimensional covalent organic frameworks. Science 2018, 361 (6397), 53.

16. Jin, S. B.; Ding, X. S.; Feng, X.; Supur, M.; Furukawa, K.; Takahashi, S.; Addicoat, M.; El-Khouly, M. E.; Nakamura, T.; Irle, S.; Fukuzumi, S.; Nagai, A.; Jiang, D. L., Charge Dynamics in A Donor-Acceptor Covalent Organic Framework with Periodically Ordered Bicontinuous Heterojunctions. Angew Chem Int Edit 2013, 52 (7), 2017-2021

17. Ding, X. S.; Guo, J.; Feng, X. A.; Honsho, Y.; Guo, J. D.; Seki, S.; Maitarad, P.; Saeki, A.; Nagase, S.; Jiang, D. L., Synthesis of Metallophthalocyanine Covalent Organic Frameworks That Exhibit High Carrier Mobility and Photoconductivity. Angew Chem Int Edit 2011, 50 (6), 1289-1293.

18. Li, H. F.; Li, H.; Xun, S. N.; Bredas, J. L., Doping Modulation of the Charge Injection Barrier between a Covalent Organic Framework Monolayer and Graphene. Chem. Mater. 2020, 32 (21), 9228-9237.

19. Ding, H. M.; Li, J.; Xie, G. H.; Lin, G. Q.; Chen, R. F.; Peng, Z. K.; Yang, C. L.; Wang, B. S.; Sun, J. L.; Wang, C., An AIEgen-based 3D covalent organic framework for white light-emitting diodes. Nature Communications 2018, 9, 5234.

20. Brixner, T.; Hildner, R.; Kohler, J.; Lambert, C.; Wurthner, F., Exciton Transport in Molecular Aggregates - From Natural Antennas to Synthetic Chromophore Systems. Adv Energy Mater 2017, 7 (16), 1700236.

21. Flanders, N. C.; Kirschner, M. S.; Kim, P.; Fauvell, T. J.; Evans, A. M.; Helweh, W.; Spencer, A. P.; Schaller, R. D.; Dichtel, W. R.; Chen, L. X., Large Exciton Diffusion Coefficients in TwoDimensional Covalent Organic Frameworks with Different Domain Sizes Revealed by Ultrafast Exciton Dynamics. J. Am. Chem. Soc. 2020, 142 (35), 14957-14965.

22. Huang, N.; Krishna, R.; Jiang, D. L., Tailor-Made Pore Surface Engineering in Covalent Organic Frameworks: Systematic Functionalization for Performance Screening. J. Am. Chem. Soc. 2015, 137 (22), 7079-7082. 
23. Wan, S.; Gandara, F.; Asano, A.; Furukawa, H.; Saeki, A.; Dey, S. K.; Liao, L.; Ambrogio, M. W.; Botros, Y. Y.; Duan, X. F.; Seki, S.; Stoddart, J. F.; Yaghi, O. M., Covalent Organic Frameworks with High Charge Carrier Mobility. Chem. Mater. 2011, 23 (18), 4094 4097.

24. Chavez, A. D.; Smith, B. J.; Smith, M. K.; Beaucage, P. A.; Northrop, B. H.; Dichtel, W. R., Discrete, Hexagonal Boronate Ester-Linked Macrocycles Related to Two-Dimensional Covalent Organic Frameworks. Chem. Mater. 2016, 28 (14), 4884-4888.

25. Chavez, A. D.; Evans, A. M.; Flanders, N. C.; Bisbey, R. P.; Vitaku, E.; Chen, L. X.; Dichtel, W. R., Equilibration of ImineLinked Polymers to Hexagonal Macrocycles Driven by Self-Assembly. Chem-Eur J 2018, 24 (16), 3989-3993.

26. Wang, S. W.; Chavez, A. D.; Thomas, S.; Li, H.; Flanders, N. C.; Sun, C.; Strauss, M. J.; Chen, L. X.; Markvoort, A. J.; Bredas, J. L.; Dichtel, W. R., Pathway Complexity in the Stacking of ImineLinked Macrocycles Related to Two-Dimensional Covalent Organic Frameworks. Chem. Mater. 2019, 31 (17), 7104-7111.

27. Xu, H.; Gao, J.; Jiang, D. L., Stable, crystalline, porous, covalent organic frameworks as a platform for chiral organocatalysts. Nat Chem 2015, 7 (11), 905-912.

28. Sun, C.; Shen, M.; Chavez, A. D.; Evans, A. M.; Liu, X L.; Harutyunyan, B.; Flanders, N. C.; Hersam, M. C.; Bedzyk, M. J.; de la Cruz, M. O.; Dichtel, W. R., High aspect ratio nanotubes assembled from macrocyclic iminium salts. P Natl Acad Sci USA 2018, 115 (36), 8883-8888.

29. Li, R. L.; Flanders, N. C.; Evans, A. M.; Ji, W.; Castano, I.; Chen, L. X.; Gianneschi, N. C.; Dichtel, W. R., Controlled growth of imine-linked two-dimensional covalent organic framework nanoparticles. Chem Sci 2019, 10 (13), 3796-3801.

30. Greenfield, S. R.; Wasielewski, M. R., Near-TransformLimited Visible and near-Ir Femtosecond Pulses from Optical Parametric Amplification Using Type-Ii Beta-Barium Borate. Opt. Lett. 1995, 20 (12), 1394-1396.

31. Hayes, D.; Kohler, L.; Hadt, R. G.; Zhang, X. Y.; Liu, C. M.; Mulfort, K. L.; Chen, L. X., Excited state electron and energy relays in supramolecular dinuclear complexes revealed by ultrafast optical and X-ray transient absorption spectroscopy. Chem Sci 2018, 9 (4), 860-875.

32. Fauvell, T. J.; Cai, Z. X.; Kirschner, M. S.; Helweh, W.; Kim, P.; Zheng, T. Y.; Schaller, R. D.; Yu, L. P.; Chen, L. X., Effects of Intra- and Interchain Interactions on Exciton Dynamics of PTB7 Revealed by Model Oligomers. Molecules 2020, 25 (10), 2441.

33. Kirschner, M. S.; Diroll, B. T.; Brumberg, A.; Leonard, A. A.; Hannah, D. C.; Chen, L. X.; Schaller, R. D., Optical Signatures of Transiently Disordered Semiconductor Nanocrystals. Acs Nano 2018, 12 (10), 10008-10015.

34. Slama-Schwok, A.; Blancharddesce, M.; Lehn, J. M., Intramolecular Charge-Transfer in Donor-Acceptor Molecules. J. Phys. Chem. 1990, 94 (10), 3894-3902.

35. Andrew, T. L.; Swager, T. M., Structure-Property Relationships for Exciton Transfer in Conjugated Polymers. J Polym Sci Pol Phys 2011, 49 (7), 476-498.

36. Belowich, M. E.; Stoddart, J. F., Dynamic imine chemistry. Chem. Soc. Rev. 2012, 41 (6), 2003-2024.

37. Haug, W. K.; Moscarello, E. M.; Wolfson, E. R.; McGrier, P. L., The luminescent and photophysical properties of covalent organic frameworks. Chem. Soc. Rev. 2020, 49 (3), 839-864.

38. Jakowetz, A. C.; Hinrichsen, T. F.; Ascherl, L.; Sick, T.; Calik, M.; Auras, F.; Medina, D. D.; Friend, R. H.; Rao, A.; Bein,
T., Excited-State Dynamics in Fully Conjugated 2D Covalent Organic Frameworks. J. Am. Chem. Soc. 2019, 141 (29), 11565-11571.

39. Spano, F. C.; Silva, C., H- and J-Aggregate Behavior in Polymeric Semiconductors. Annual Review of Physical Chemistry, Vol 65 2014, 65, 477-500.

40. Dufresne, S.; Bourgeaux, M.; Skene, W. G., Tunable spectroscopic and electrochemical properties of conjugated push-push, push-pull and pull-pull thiopheno azomethines. J. Mater. Chem. 2007, 17 (12), 1166-1177.

41. Bourgeaux, M.; Guarin, S. A. P.; Skene, W. G., Photophysical, crystallographic, and electrochemical characterization of novel conjugated thiopheno azomethines. J. Mater. Chem. 2007, 17 (10), 972-979.

42. Takeuchi, S.; Ruhman, S.; Tsuneda, T.; Chiba, M.; Taketsugu, T.; Tahara, T., Spectroscopic Tracking of Structural Evolution in Ultrafast Stilbene Photoisomerization. Science 2008, 322 (5904), 1073-1077.

43. Lim, E. C., Proximity Effect in Molecular Photophysics Dynamic Consequences of Pseudo-Jahn-Teller Interaction. J. Phys. Chem. 1986, 90 (26), 6770-6777.

44. Quenneville, J.; Martinez, T. J., Ab initio study of cis-trans photoisomerization in stilbene and ethylene. J. Phys. Chem. A 2003, 107 (6), 829-837.

45. Bourgeaux, M.; Skene, W. G., Photophysics and electrochemistry of conjugated oligothiophenes prepared by using azomethine connections. J. Org. Chem. 2007, 72 (23), 8882-8892.

46. Wei, Z. W.; Gu, Z. Y.; Arvapally, R. K.; Chen, Y. P.; McDougald, R. N.; Ivy, J. F.; Yakovenko, A. A.; Feng, D. W.; Omary, M. A.; Zhou, H. C., Rigidifying Fluorescent Linkers by MetalOrganic Framework Formation for Fluorescence Blue Shift and Quantum Yield Enhancement. J. Am. Chem. Soc. 2014, 136 (23), 82698276 .

47. Nogueira, J. J.; Plasser, F.; Gonzalez, L., Electronic delocalization, charge transfer and hypochromism in the UV absorption spectrum of polyadenine unravelled by multiscale computations and quantitative wavefunction analysis. Chem Sci 2017, 8 (8), 5682-5691.

48. Ghosh, R.; Pochas, C. M.; Spano, F. C., Polaron Delocalization in Conjugated Polymer Films. J Phys Chem C 2016, 120 (21), 11394-11406.

49. Trinkunas, G.; Herek, J. L.; Polivka, T.; Sundstrom, V.; Pullerits, T., Exciton delocalization probed by excitation annihilation in the light-harvesting antenna LH2. Phys. Rev. Lett. 2001, 86 (18), 4167-4170.

50. Yoon, M. C.; Cho, S.; Kim, P.; Hori, T.; Aratani, N.; Osuka, A.; Kim, D., Structural Dependence on Excitation Energy Migration Processes in Artificial Light Harvesting Cyclic Zinc(II) Porphyrin Arrays. J. Phys. Chem. B 2009, 113 (45), 15074-15082.

51. Schlosser, F.; Sung, J.; Kim, P.; Kim, D.; Wurthner, F., Excitation energy migration in covalently linked perylene bisimide macrocycles. Chem Sci 2012, 3 (9), 2778-2785.

52. Bradforth, S. E.; Jimenez, R.; Vanmourik, F.; Vangrondelle, R.; Fleming, G. R., Excitation Transfer in the Core Light-Harvesting Complex (Lh-1) of Rhodobacter-Sphaeroides - an Ultrafast Fluorescence Depolarization and Annihilation Study. J. Phys. Chem. 1995, 99 (43), 16179-16191. 


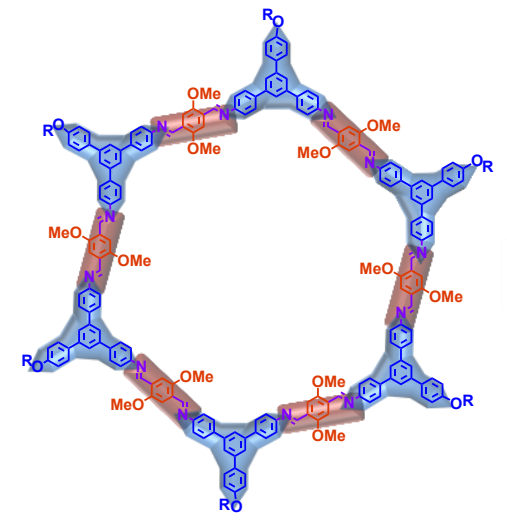

Rapid Excited State Decay

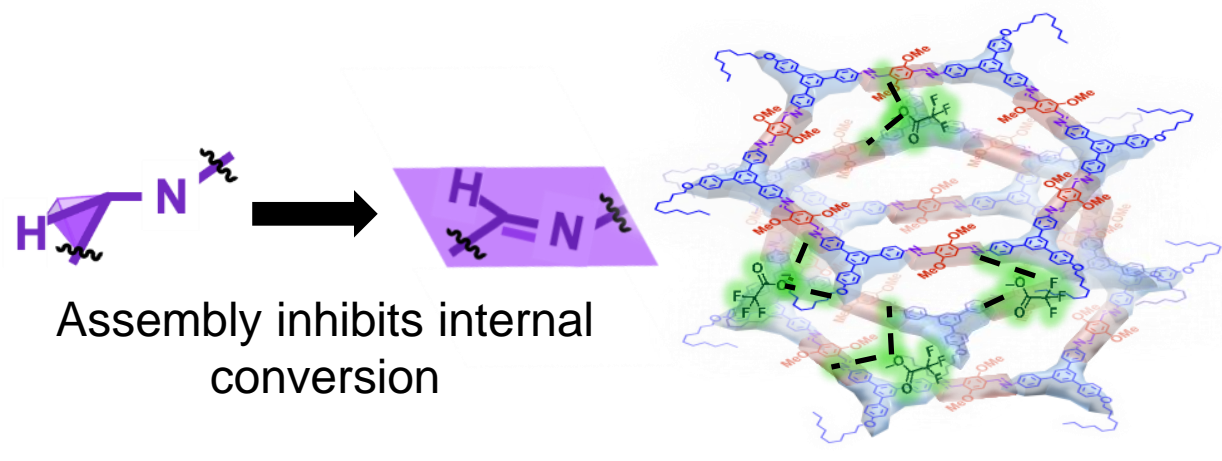

Increased Exciton Lifetime 